\section{Issues in Survey Development: Assumption Checking, Behavior Coding, Cognitive Interviewing, and Design Testing}

Joe Chelladurai

\begin{abstract}
Survey research can benefit from pretesting and greatly enhance the effectiveness of data collection and analytic processes. For this purpose, a comprehensive process involving four aspects of testing is proposed, namely, assumption checking, behavior coding, cognitive interviewing, and design testing. In this article, I briefly explain each aspect with relevant illustrations.
\end{abstract}

Keywords: survey development, behavior coding, cognitive interviewing, design testing

When researchers and methodologists design their studies, a vital part is the collection of data. Although theoretical and methodological frameworks may be demonstrated in the study design and how findings are communicated, there is an important need to examine the survey instruments and their delivery and response in particular. To examine this issue, a comprehensive and reflective examination of four aspects of survey development is proposed which involves assumption checking, behavior coding, cognitive interviewing, and design testing. These aspects are iterative and cyclical and can be used in the survey design, pretesting, and data analytic stages. Feedback on measurement instruments can provide important insights for ongoing and future development of surveys.

\section{Assumption Checking}

A major assumption of survey research is standardization, which relates to whether the nature of questions and responses have the same meaning across groups. To check for major and minor assumptions, survey development may include reflective questions that guide question wording and response options. Questions may include: Are my assumptions culture specific? Do survey questions have the same meaning when translated? Do I assume that all respondents are comfortable being asked certain questions? If so, are there response options that reflect their answers.

Survey questions may be worded by researchers based on their own familiar contexts. For example, Willis et al., (2008) observed that the phrase "in your entire life" had different cultural meanings. For English respondents the phrase meant past behavior, whereas for Chinese respondents the phrase included past and future behavior. As a result, Chinese respondents had more trouble understanding the question.

Assumptions about respondent burden are important when considering the content, design, and delivery of the survey. Research findings related with respondent burden provide some valuable insights on what contributes to or lessens burden. Sharp and Frankel (1983) examined whether length, effort, and repeat administration were related with response burden, and found that only length was significantly correlated. Findings from their study also suggest that attitudinal factors such as usefulness of the survey, and concerns over character-type questions may be related with respondent burden.

\section{Behavior Coding}

Behavior coding involves examining overt indicative behaviors of interviewers and respondents. For instance, interviewer behavior can be assessed to see whether the interviewer read the complete question or whether they read only part of the question. Respondent behaviors are coded when they interrupt the interviewer, indicate that they do not understand the question, or refuse to answer.

Behavior coding typically results in quantitative data with reports indicating what percentage of participants had asked for clarification or gave inadequate or irrelevant responses. Behavior coding is achieved without interference of the researcher and without participant's awareness that their behaviors are coded when they respond to survey questions. As a result, behavior coding is a passive approach. For more active involvement from the participants, researchers may use cognitive interviewing as a follow-up procedure to know what participants experienced cognitively while taking the survey.

\section{Cognitive Interviewing}

The goals of cognitive pre-testing are to know whether respondents (a) can comprehend the logical structure of the question, (b) able to understand the question consistently, and (c) answer in line with researchers' intent (Collins, 2003). Based in theory, there are generally four actions in the question-and-answer model. These are comprehension, response, judgement, and retrieval. These four processes are interwoven and often occur in iterations than linear sequences.

Against the backdrop of the question-and-answer model, researchers may employ two varying methods of cognitive interviewing, namely, think aloud interviewing and probing. In think-aloud interviewing, the respondent does most of the talking and states how they went about answering questions, what they were thinking, and talk about any hesitations they encountered. They may also talk about whether the questions were easy or difficult. On the other hand, the probing method is interviewer driven and the 
respondent answers question based on the interviewer's observations. There can be a balance of both think-aloud and probing interviewing based on the needs and stages of the pretesting.

The major drawbacks of testing surveys using cognitive interviews is the subjective nature of the undertaking. For both the interviewer and the respondent, interviewing is an experiential process and prone to subjective bias. Cognitive interviewing can account for subjectivity by examining the process itself with a meta examination of the testing stage.

\section{Design Testing}

Design testing can be very helpful in addressing respondent burden or cognitive fatigue. In web-based surveys, visual indicators such as a progress bar to indicate how long the survey might take may help respondents avoid frustration over the unknown length of the survey.

Anonymous designs such as using web-based programs versus in-person interviews have their own drawbacks. Even with anonymity offered by web-based surveys, online and digital privacy concerns may inhibit participant responses. Further, when participants hold loyalties to the organization conducting the study or perceive that there may be consequences to their responses may be concerned and thereby change the way they respond.

Design testing can include the response time of the complete survey as well as each survey question. This can help researchers with an estimate of typical time taken to respond to the survey and flag responses with may be guessed by participants who rush, as well as to learn the patterns of thoughtful respondents. Feedback at the end of the survey may also be helpful to improve survey delivery.

\section{Conclusion}

In summary, the iterative processes of assumption checking, behavior coding, cognitive interviewing, and design testing offers scope to greatly enhance survey development. With more attention to these procedures, researchers can develop high quality measurements that aid in providing robust findings. It is therefore important, and perhaps essential, for researchers to be continually trained in these aspects of survey development in order to conduct thorough and methodologically rigorous research.

\section{References}

Collins, D. (2003). Pretesting survey instruments: an overview of cognitive methods. Quality of life research, 12(3), 229-238.

Sharp, L. M., \& Frankel, J. (1983). Respondent burden: A test of some common assumptions. Public Opinion Quarterly, 47(1), 36-53.
Willis, G., Lawrence, D., Hartman, A., Kudela, M. S., Levin, K., \& Forsyth, B. (2008). Translation of a tobacco survey into Spanish and Asian languages: the Tobacco Use Supplement to the Current Population Survey. Nicotine \& tobacco research, 10(6), 10751084. 